SCIENTIFIC PUBLICATIONS OF THE STATE UNIVERSITY OF NOVI PAZAR

SER. A: Appl. Math. Inform. AND Mech. vol. 11, 1 (2019), 33-40.

\title{
A Design of an Ultra Low-Power Operational Transconductance Amplifier
}

\author{
G. S Jovanović, M. K. Stojčev, S. D. Vučković
}

\begin{abstract}
This paper describes a design of an ultra low-power, low-voltage operational transconductance amplifier (OTA). A two stage OTA is implemented in $0.13 \mu \mathrm{m} \mathrm{SiGe} \mathrm{BiCMOS} \mathrm{tech-}$ nology. The OTA operates with all transistors active in subthreshold region. Under the typical operating conditions, circuit supply voltage is $0.5 \mathrm{~V}$, supply current $150 \mathrm{nA}$ and power consumption is $75 \mathrm{nW}$. Low-frequency gain is $53 \mathrm{~dB}$, gain-bandwidth product (GBW) $350 \mathrm{kHz}$ and phase margin $55^{\circ}$. $-1 \mathrm{~dB}$ gain compression point is $-7.4 \mathrm{dBm}$ and output intercept point (OIP3) $-21.5 \mathrm{dBm}$. OTA layout active chip area is $0.0014 \mathrm{~mm}^{2}$.
\end{abstract}

Keywords: Subthreshold, weak inversion, operational transconductance amplifier, low-power.

\section{Introduction}

The interest for low-voltage power suppling and low-power consumption of analog and digital circuits has grown rapidly [1]. This increased interest is mainly due to commercial popularity of battery-powered portable equipment, which reduce the power consumption is of paramount importance. Some low-voltage/low-power consumption systems we standardly meet in laptop/notebook/tablet computers, cellular phones, implantable devices, medical electronics (pacemakers, hearing aids, blood flow meters...), multimedia and wireless devices, etc. [2].

Operational Amplifier (OPA) and Operational Transconductance Amplifier (OTA) are basic building elements of most analog circuits. Design of low-voltage/low-power OPA/OTA is crucial step during the design of such analog systems. In addition, efficient power consumption, small layout area and the compatibility in interfacing with digital circuits is imperative.

Manuscript received January 27, 2019; accepted May 11, 2019.

G. S Jovanović and M. K. Stojčev are with the Faculty of Electronic Engineering, Niš, Serbia; S. D. Vučković is with the Telekom Srbija a.d., Beograd, Serbia. 
MOS transistor in weak inversion operation has gate-source voltage smaller than the threshold voltage, as well as much larger transconductance due to exponential currentvoltage relationship [3-6]. Therefore, we chose operating points for transistors in weak inversion region with aim to obtain compact OPA/OTA circuit with minimum supply voltage. However, the impedance matching and noise property of weak inversion operation becomes worse. Thereby careful selection of transistors in weak inversion operation is important [6].

Section 2 discusses the transistors that operate in weak inversion mode. The scheme and circuits description of two stages subthreshold OTA is presented is Section 3. Section 4 reveals results of DC, AC, transient and harmonic balance simulation. The final Section 5 , conclusion, describes the contribution.

\section{Subthreshold circuits design}

A MOSFET model includes gradual change of a charge in an inversion layer between the source and drain due to the fact that the channel voltage varies from source voltage to drain voltage [7-9]. A MOSFET can be operate in one of three different modes, depending on the voltages at the terminals. The three operational modes are: weak, moderate, and strong inversion. The boundaries between the modes are not strictly defined. The approximate values are:

1. $V_{G S}>V_{T}+100 \mathrm{mV}$ - strong inversion,

2. $V_{T}+100 \mathrm{mV}>V_{G S}>V_{T}-100 \mathrm{mV}$ - moderate inversion,

3. $V_{G S}<V_{T}-100 \mathrm{mV}$ - weak inversion.

In strong inversion region, variation of drain current in terms of gate-to-source voltage is given by

$$
I_{D}=\frac{\mu C_{o x}}{2} \frac{W}{L}\left(V_{G S}-V_{T}\right)^{2}\left[1+\lambda\left(V_{D S}-V_{D S P}\right)\right]
$$

where $V_{T}$ is the nominal threshold voltage, $\lambda$ is the channel length modulation factor, and $V_{D S P}$ is the drain-to-source pinch-off voltage.

The strong inversion MOSFET model assumes that the drain current, $I_{D}$, falls to zero when the gate voltage drops below the threshold voltage $V_{T}$. However, this is not quite true. In weak inversion, the number of free carriers in the channel is small enough to lead to negligible drift current, but diffusion current makes the MOSFET to operate more like a bipolar junction transistor. Below threshold, the channel charge drops exponentially by decreasing the gate voltage [3-6]. The relation between drain current and gate-to-source voltage in weak inversion region is given by

$$
I_{D}=I_{D 0} \frac{W}{L} e^{\frac{q V_{G S}}{n k T}}
$$




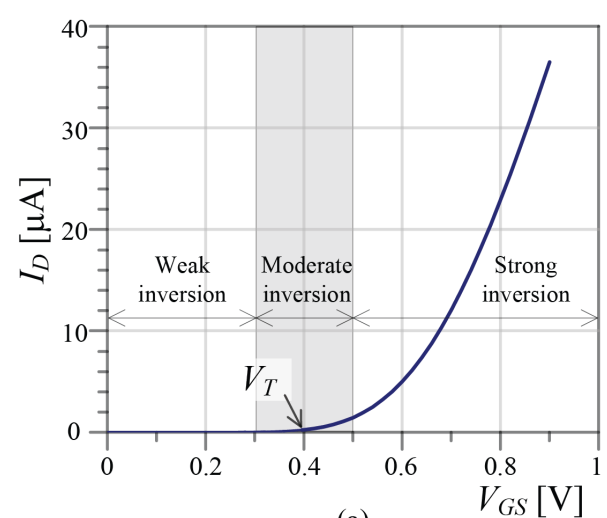

(a)

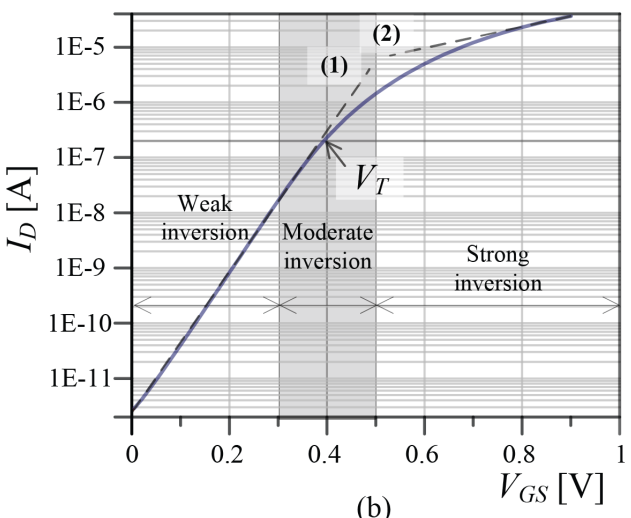

(b)

Fig. 1. MOS transistor transfer characteristics in weak, moderate and strong inversion as (a) lin-lin presentation, (b) lin-log presentation.

where $n$ and $I_{D 0}$ can be extracted from measured transfer characteristics of the transistor.

Fig. 1 shows the transfer MOSFET characteristic curve, $I_{D}=f\left(V_{G S}\right)$. The threshold voltage, $V_{T}$, is around $0.4 \mathrm{~V}$. Fig. 1(a) presents characteristic on standard linear-linear axis plot. Fig. 1(b) shows the same characteristic, but drain current is now presented by using logarithmic $y$ axis scale. The figure clearly shows that the slope (1) of the exponential weak inversion curve is significantly higher than the slope (2) of the quadratic strong inversion curve.

MOSFET dynamic performances, which operates in subthreshold region, can be evaluated from the equitation (2). Transistor transconductance is

$$
g_{m}=\frac{\partial I_{D}}{\partial V_{G S}}=\frac{I_{D}}{n \cdot \frac{k T}{q}}
$$

and it linearly depends on the drain bias current. The channel-length modulation effect is identical for weak and strong inversion. The variation of drain-source resistance in terms of drain current is inverse in both regions, that is

$$
r_{d}=\frac{\partial I_{D}}{\partial V_{D S}}=\frac{1}{\lambda \cdot I_{D}}
$$

For common-source single stage amplifier the low-frequency voltage gain is

$$
A_{v}=g_{m} \cdot r_{d}
$$

\section{Two-stage OTA operating in weak inversion}

Fig. 2 shows the electrical scheme of a two stages transconductance amplifier. The first stage uses differential amplifier with $n$-channel transistors at the input. The second stage is 


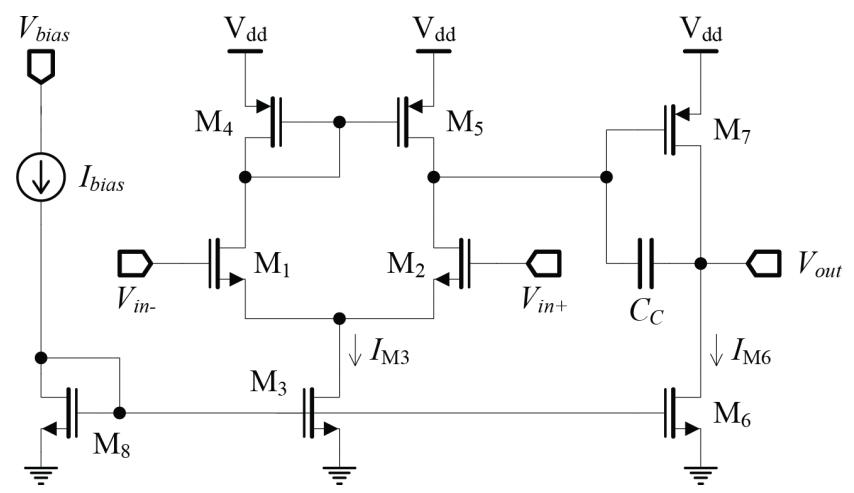

Fig. 2. Scheme of CMOS two stage OTA in weak inversion

conventional inverter with active load. The same reference current is used for the differential amplifier and inverter stage. Bias currents in the both stages are controlled together.

In the differential amplifier, composed of transistors $\mathrm{M}_{1}$ and $\mathrm{M}_{2}$, conversion from differential input to single ended output is achieved by using current mirror, transistors $\mathrm{M}_{4}$ and $M_{5}$. The current of $M_{1}$ is mirrored by $M_{4}$ and $M_{5}$ and subtracted from the $M_{2}$ current.The result current on the output resistance of the first stage gives the single-ended output voltage. Final, the output signal, $V_{\text {out }}$, is obtained with second stage. Capacitor $C_{C}$ takes care of compensation requirements.

In order to obtain higher voltage gain, it is necessary to increase the transconductance or to augment the output resistance. The output resistance can be augmented by making the transistor longer $(L)$. The transconductance can be increased by raising the drain current, but this decreases the output resistance, so this is not helpful. If a transistor is operating in strong inversion, it is possible to increase the transconductance by increasing $W / L$. If a transistor is operating in weak inversion region, the transconductance is independent of $W / L$, so there is no way to increase it without lowering the output resistance.

Taking into account the expressions of $g_{m}$ and $r_{d}$, defined in equitation (3) and (4), the DC gain of the two-stage OTA can be approximately written as follows:

$$
A_{v}=g_{m 2} \frac{r_{d 2} r_{d 5}}{r_{d 2}+r_{d 5}} \cdot g_{m 7} \frac{r_{d 6} r_{d 7}}{r_{d 6}+r_{d 7}}=\frac{1}{n_{2} \cdot n_{6}\left(\frac{k T}{q}\right)^{2}} \frac{1}{\left(\lambda_{2}+\lambda_{5}\right)\left(\lambda_{6}+\lambda_{7}\right)} .
$$

Based on the equation (6), gain in weak inversion region is independent of bias current and $W / L$ ratio. The typical resistance of the nodes in weak inversion varies from several tens of $\mathrm{M} \Omega$ up to G $\Omega$. This means that the poles of the amplifier transfer characteristics are very low and the bandwidth is limited. The higher bias current will provide greater transconductance and increase bandwidth.

If transconductance $g_{m 2}$ is fixed and the compensation capacitor $C_{C}$ should be defined 
according to the following equation in order to achieve the desired gain-bandwidth product (GBW):

$$
G B W=\frac{g_{m 2}}{2 \pi C_{C}}
$$

\section{Simulation results}

For design simulation and verification of the proposed circuit we used the IHP design kit SG13S, for $0.13 \mu \mathrm{m}$ BiCMOS technology, and the electronic design software Advanced Design System (ADS) version 2014.01 [10].

The design procedure starts from a constraint which relate to DC operating point, i.e., by choosing bias currents $I_{M 3}$ and $I_{M 6}$. Selected values are $I_{M 3}=50 \mathrm{nA}$ and $I_{M 6}=100 \mathrm{nA}$, what means that the total OTA's supply current is $I_{D C}=150 \mathrm{nA}$. Power supply voltage of $0.5 \mathrm{~V}$ was used, and power consumption of $75 \mathrm{nW}$ is obtained. Fig. 3. shows OTA's DC characteristics: outputs voltages and supply current in term of differential input voltage.

Fig. 4 shows a frequency response of the OTA. The low-frequency voltage gain is 53 $\mathrm{dB}$, unity-gain bandwidth (GBW) is $350 \mathrm{kHz}$, and phase margin is $55^{\circ}$.

The $I_{M 6}$ bias current is set to increase the OTA slew rate (SR), and is given by

$$
S R=\frac{I_{M 6}}{C_{C}+C_{L}}
$$

where $C_{L}$ is output load capacitance. For load and compensation capacitors, $C_{L}=1 \mathrm{pF}$ and $C_{C}=350 \mathrm{fF}$ respectively, simulation result shows that the slew rate is $0.105 \mathrm{~V} / \mu \mathrm{S}$.

OTA's nonlinear characteristics were tested. The first test relates to $1 \mathrm{kHz}$ single tone input. $1 \mathrm{~dB}$ compression point was determined, and it corresponds to $\mathrm{P}_{-1 d B}=-7.4 \mathrm{dBm}$. Simulation results are presented in Fig. 5.

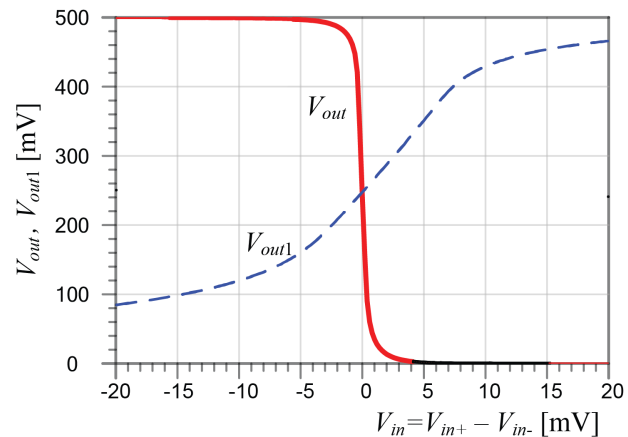

(a)

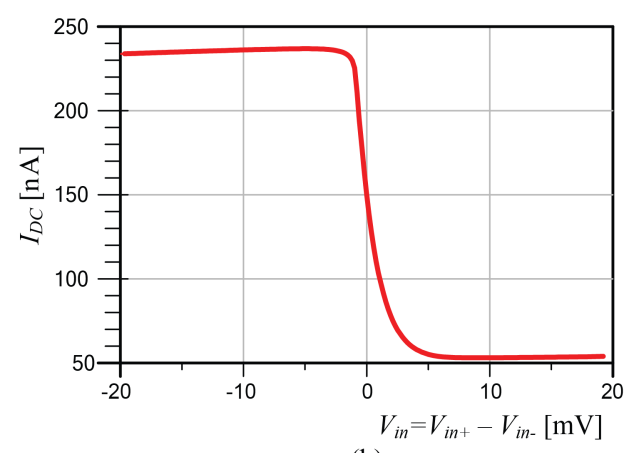

(b)

Fig. 3. OTA's DC characteristics in weak inversion (a) outputs voltages and (b) supply current in term of input voltage. 


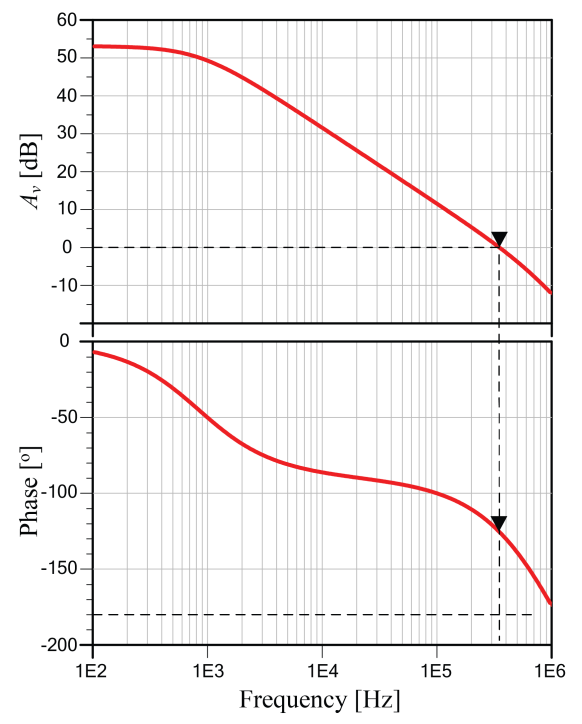

Fig. 4. OTAs AC characteristic: voltage gain and phase shift in term of frequency.

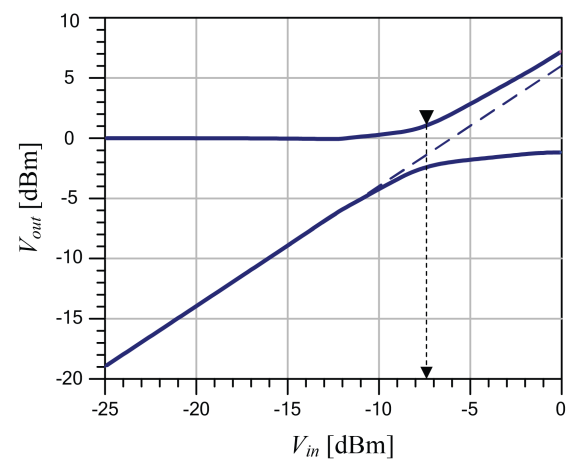

Fig. 5. OTA's $-1 \mathrm{~dB}$ compression point.

Total harmonic distortion (THD) is simulated at frequency of $1 \mathrm{kHz}$ and $100 \mathrm{mV}$ output voltage amplitude. Outputwaveform distortion relative to input pure sine wave is $-41 \mathrm{~dB}$.

Next, at $1 \mathrm{kHz}$ frequency, the OTA was tested with two tones input signal, at $100 \mathrm{~Hz}$ frequency spacing. The output intercept point OIP3 $=-21.4 \mathrm{dBm}$ was determined by applying harmonic balance analysis.

The summary of the two stage subthreshold operational transconductance amplifier characteristics are given in Table 1.

The OTA chip layout drawing is shown in Figure 6. Active area occupation is just 40 * $35=1400 \mu \mathrm{m}^{2}$. 
Table 1. Summary of amplifier performances.

\begin{tabular}{|l|l|}
\hline Parameters & Values \\
\hline Process & $0.13 \mu \mathrm{m}$ \\
\hline Supply voltage & $0.5 \mathrm{~V}$ \\
\hline DC supply current & $150 \mathrm{nA}$ \\
\hline Power & $75 \mathrm{nW}$ \\
\hline GBW gain bandwidth & $350 \mathrm{kHZ}$ \\
\hline Phase margin & $55^{\circ}$ \\
\hline DC gain & $53 \mathrm{~dB}$ \\
\hline Slew Rate & $0.105 \mathrm{~V} / \mu \mathrm{S}$ \\
\hline$-1 \mathrm{~dB}$ compression point & $-7.4 \mathrm{dBm}$ \\
\hline IIP3 & $-21.5 \mathrm{dBm}$ \\
\hline THD @ $1 \mathrm{kHz}, V_{\text {out }}=100 \mathrm{mV}$ & $-41 \mathrm{~dB}$ \\
\hline Silicon area & $0.0014 \mathrm{~mm}^{2}$ \\
\hline
\end{tabular}

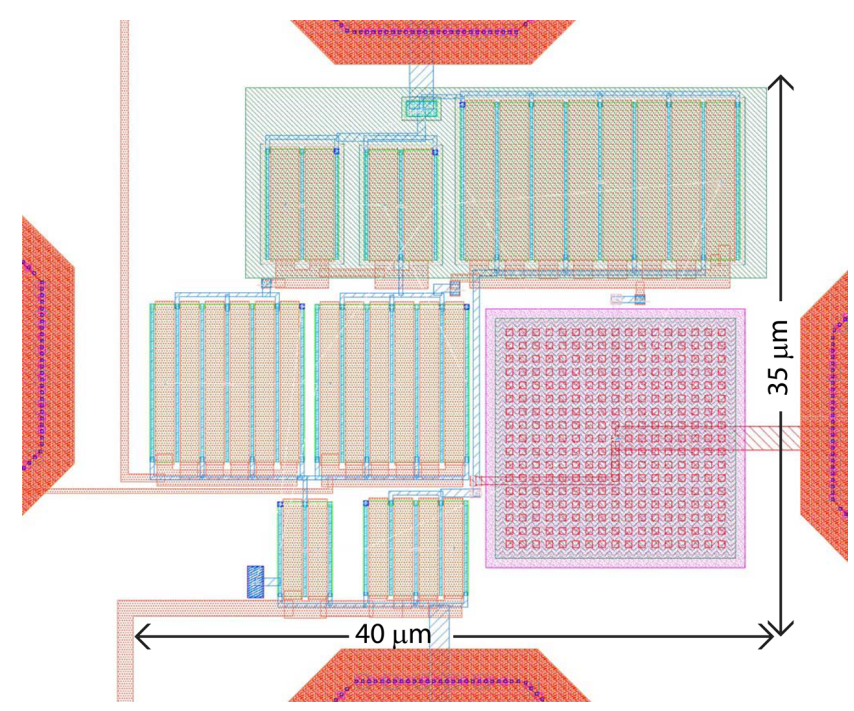

Fig. 6. OTA chip layout drawing.

\section{Conclusion}

Design of low-voltage ultra low-power subthreshold operational trasconductance amplifier is presented. The capability of subthreshold OTA implemented as two-stage amplifier was tested in a $0.13 \mu \mathrm{m}$ BiCMOS technology. The OTAwas supplied with $0.5 \mathrm{~V}$. Under this condition a low-frequency gain of $53 \mathrm{~dB}$ was achieved. $\mathrm{GBW}$ is $350 \mathrm{kHz}$ with power consumption of only $75 \mathrm{nW}$. 
This design demonstrates that carefully-designed subthreshold OTA represents a good candidate solution which can be used in low-voltage, low-power electronic building block that we meet in analog section of mixed-signal integrated circuits.

\section{References}

[1] O. Abdelfattah, G. W. Roberts, I. Shin, Y. Shih, An Ultra-Low-Voltage CMOS Process-Insensitive Self-Biased OTA with Rail-to-Rail Input Range, IEEE Transactions on Circuits and Systems-I: Regular Papers, Vol. 62, No. 10, (2015), 2380-2390.

[2] M. Borhani, F. RaZaghian, Low Power Op-Amp Based on Weak Inversion with MillerCascoded Frequency Compensation, International Multi Conference of Engineers and Computer Scientists 2010, Vol II, Hong Kong, 2010, pp. 1413-1417.

[3] D. J. Comer, D. T. Comer, Using the Weak Inversion Region to Optimize Input Stage Design of CMOS Op Amps, IEEE Transactions on circuits and systems - II: Express briefs, Vol. 51, No. 1 (2004), 8-14.

[4] C. C. ENZ, F. KRUMmenacher, E. A. Vittoz, An analytical MOS transistor model valid in all regions of operation and dedicated to low-voltage and low-current applications, Analog Integrated Circuits and Signal Processing, Vol. 8, No. 1 (1995), 83-114.

[5] A. Wang, B. H. Calhoun, A. P. Chandrakasan, Sub-threshold Design for Ultra LowPower Systems, Springer, New York, 2006.

[6] L. Magnelli, F. A. Amoroso, F. Crupi, G. Cappuccino, G. Iannaccone, Design of a 75- $n W, 0.5-V$ subthreshold complementary metal-oxide-semiconductor operational amplifier, International Journal of Circuit Theory and Applications, Vol. 42, No. 9 (2014), 967-977.

[7] M. O'Halloran, R. SarpeshKar, A 10-nW 12-bit Accurate Analog Storage Cell with 10-aA Leakage, IEEE Journal of Solid-State Circuits, Vol. 39, No. 11 (2004), 1985-1996.

[8] E. Vittoz, J. Fellrath, "CMOS Analog Integrated Circuits Based on Weak Inversion Operation", IEEE Journal of Solid-State Circuits, Vol. 12, No. 3 (1977), 224-231.

[9] A. Tajalli, Y. Leblebici, Extreme Low-Power Mixed Signal IC Design: Subthreshold Source-Coupled Circuits, Springer, New York, 2010.

[10] IHP-Microelectronics, SiGe:C BiCMOS Technologies for MPW \& Prototyping, http://www.ihp-microelectronics.com/en/services/mpw-prototyping/sigec-bicmostechnologies.html, available at April, 2019. 